\title{
Rôle et intérêt de zones d'écrêtement des crues
}

\author{
par O. Gilard \\ Cemagref, Division Hydrologie-Hydraulique, Lyon
}

\section{INTRODUCTION}

Il n'est sans doute pas nécessaire de rappeler les dernières crues aux conséquences catastrophiques qui ont touché la France ces dernières années pour introduire cet article. Sur un plan purement économique, ce ne sont d'ailleurs pas les plus médiatisées (Nîmes, 1988; Vaison la Romaine, 1992; Puyserguier, 1996) qui ont coûté le plus cher à la nation mais ce sont celles généralisées dans le nord et l'est de la France au cours de I'hiver 1994-95. Rappelons aussi que le bassin de la Loire n'a pas été touché récemment, la dernière grande crue remontant à 1982. La crue précédente de 1980 ayant causé la catastrophe de Brives-Charensac était limitée au haut bassin. Cependant la probabilité d'un événement majeur sur ce fleuve reste la même : faible mais non nulle !

Ces événements ont amené l'ensemble des personnes concernées (scientifiques. techniciens, politiques, citoyens) à réfléchir à de nouveaux moyens de prendre en compte ce problème pour en diminuer les conséquences, et, en particulier, à préconiser la remise en service de larges zones d'épandages de crue. Nous allons tenter, après quelques rappels sur les enjeux de l'aménagement des cours d'eau et sur le risque d'inondation, de montrer en quoi ces zones peuvent rendre des services considérables tant pour diminuer les dommages dus aux inondations que pour préserver une ressource en eau de plus en plus sollicitée et un environnement de qualité. L'objectif poursuivi est de définir les moyens d'une mise en valeur « durable» de ces zones conciliant les différents enjeux qui y sont liés. Les progrès scientifiques en hydrologie, hydraulique, cartographie,... de ces dernières années donnent aujourd'hui les moyens de les analyser sur des bases objectives.

\section{II. 圆 RAPPELS}

\subsection{Les enjeux de l'aménagement des rivières}

Avant d'entrer au cœur du sujet, il parait important de rappeler que, même si l'attention se porte surtout sur une problématique de gestion du risque (d'inondation), une rivière, et l'eau qui y coule, présentent bien d'autres enjeux tout aussi importants pour la collectivité. Le premier d'entre eux reste

\section{Flood cutting down areas role and interest}

There are many stakes linked with river management and especially with Loire river: water ressource, environment, flood risk are the three main types. Flood plain areas may play an important function to satisfy part of these stakes. Their hydraulic functioning allow flood lamination and favour indirectly water ressource both in quantity and quality. They may keep an economic interest by productive activities which tolerate flood constraint or, indirectly, by leasure activities. At least, they have an essential role for environment due to specific water ecosystems linked to them. The limits in the efficiency of classical works and social demand evolutions lead to find new management rules sumarised in the "Dynamic slow-down" concept. New methods like Inondability allows their effective uses to take into account the diversified stakes and situations along a river. Applied to Loire river, this evolution should favour a more harmonious development of the valley and a reduction of flood damages in case of exceptional flow, that, if it occures today, would have dramatic consequences. 


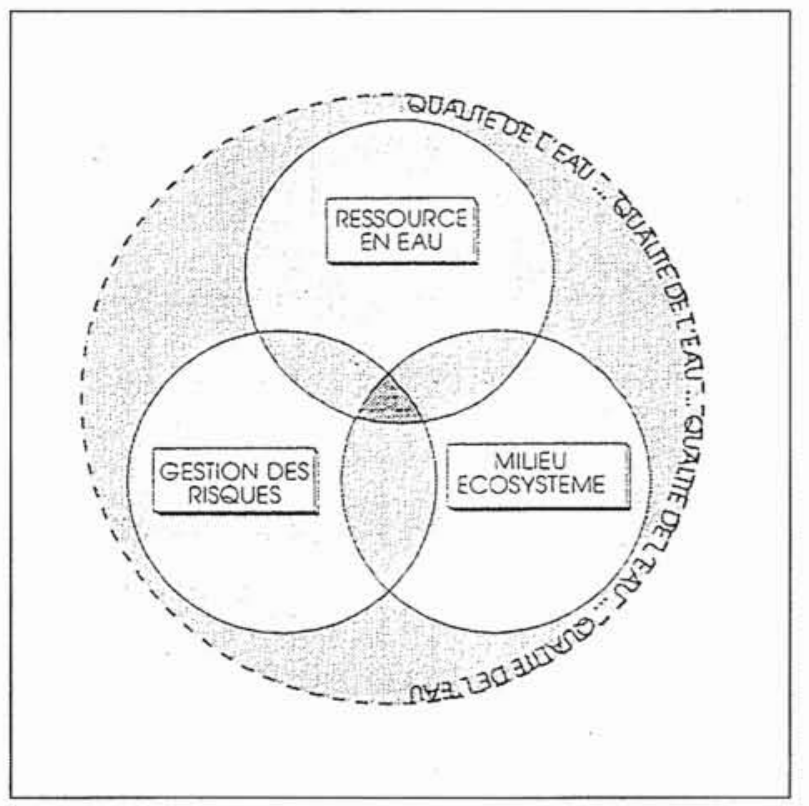

1. Les enjeux de l'aménagement des rivières.

bien entendu la ressource en eau, nécessaire à toutes les activités domestiques et économiques (de l'eau potable à l'eau d'irrigation en passant par l'eau industrielle).

Le second enjeu est représenté par l'environnement associé à ce système naturel complexe que représente le cours d'eau et les zones connexes qui y sont liées. Habitat d'une faune aquatique riche, permettant les développements d'une végétation typique (ripisylve et forêts alluviales) elle-même hôte d'une faune et d'une flore intéressantes, cet environnement permet aujourd'hui l'accueil de nombreuses activités de loisirs de plus en plus indispensables à l'homme, citadin en particulier, à la recherche de zones récréatives de qualité.

Le troisième enjeu consiste à développer des activités économiques classiques pour valoriser les zones de bordure de cours d'eau: urbanisation, zones d'activité, agriculture, puisqu'elles présentent en général des qualités attractives dues aux caractéristiques des terrains qui s'y trouvent (facilité d'accès et de desserte, fertilité,...). A cet enjeu est lié le problème du risque d'inondation dont la manifestation potentielle menace la permanence de ces usages, voire leur rentabilité. Ces différents enjeux sont illustrés par la figure 1 .

Tout au long du cours de la Loire ces diverses préoccupations se retrouvent :

- une présence humaine dense le long de la vallée nécessitant une alimentation en eau pour l'ensemble de ses activités ;

- une pression environnementale forte soutenue par de nombreuses associations pour la sauvegarde d'un écosystème qui apparaît à maints égards comme encore «sauvage $»$;

- un fort besoin de développement en particulier de certains centres urbains importants comme Orléans ou Tours.

La demande de plus en plus pressante d'une réelle prise en compte des multiples enjeux liés à ce fleuve se traduit par la mise en place de différents projets comme le Plan Loire, pour arbitrer les conflits d'usage qui existent et maîtriser l'évolution du système.

\subsection{Le risque d'inondation}

Le risque d'inondation lui-même résulte d'une réalité complexe dans laquelle deux facteurs interviennent plus ou moins indépendamment l'un de l'autre. Selon une définition à peu près reconnue par tous, le premier d'entre eux est dénommé «aléa » et traduit la contrainte hydraulique liée au fonctionnement hydraulique de la rivière et hydrologique de son bassin versant. Le second, dénommé « vulnérabilité », traduit la nature socio-économique du problème : en effet, il ne suffit pas qu'un cours d'eau déborde pour être confronté à un risque d'inondation ; encore faut-il qu'il déborde à un endroit où cela pose problème, c'est-à-dire un endroit vulnérable aux inondations. Seule la combinaison de ces deux facteurs permet de déterminer une situation à "risque " avec une acuité variable [1] (fig. 2). Classiquement, la tendance est de s'intéresser surtout au facteur hydraulique (l'aléa), ce qui a conduit aux types d'aménagements, parfois qualifiés de structurels, que nous connaissons bien (endiguements, recalibrages, barrages). Malheureusement, l'accroissement parfois déraisonné de la vulnérabilité à l'abri (relatif) de ces aménagements peut conduire in fine à une augmentation du risque, et donc à l'inverse du but initialement recherché. Aujourd'hui, l'évolution de la société et les limites d'efficacité atteintes par ces aménagements traditionnels rendent nécessaire de revoir les pratiques d'aménagement de l'espace autour des rivières et de maîtrise de la vulnérabilité, en particulier dans les zones dites " d'épandage de crue " qui sont, de fait, des zones où l'on cherche à maintenir une vulnérabilité faible pour conserver leur caractère inondable et leur faire jouer un rôle hydraulique (entre autres) que nous analyserons un peu plus en détail ci-après. Cela passe par l'acceptation d'un certain niveau de risque puisque tout le monde s'accorde aujourd'hui sur l'impossibilité de la maîtrise totale du risque, que traduit l'expression "le risque zéro n'existe pas! ».

Ces différentes évolutions existent sur le bassin de la Loire où des aménagements hydrauliques ont été mis en œuvre depuis fort longtemps (digues remontant à l'époque des Plantagenêts, $\mathrm{XII}^{\mathrm{C}}$ siècle) et où. dès le $\mathrm{XVIII}^{\mathrm{c}}$ siècle, on s'était rendu compte des limites d'une solution « tout endiguement» [2]. Le long de ce fleuve apparaissent aujourd'hui des enjeux forts tant en terme de maîtrise du risque que de préservation d'un environnement reconnu pour son originalité. Les conflits d'usage sur certaines zones réputées inondables sont bien connus et il est urgent de trouver des solutions pour anticiper et réduire les conséquences catastrophiques que pourrait avoir une crue exceptionnelle.

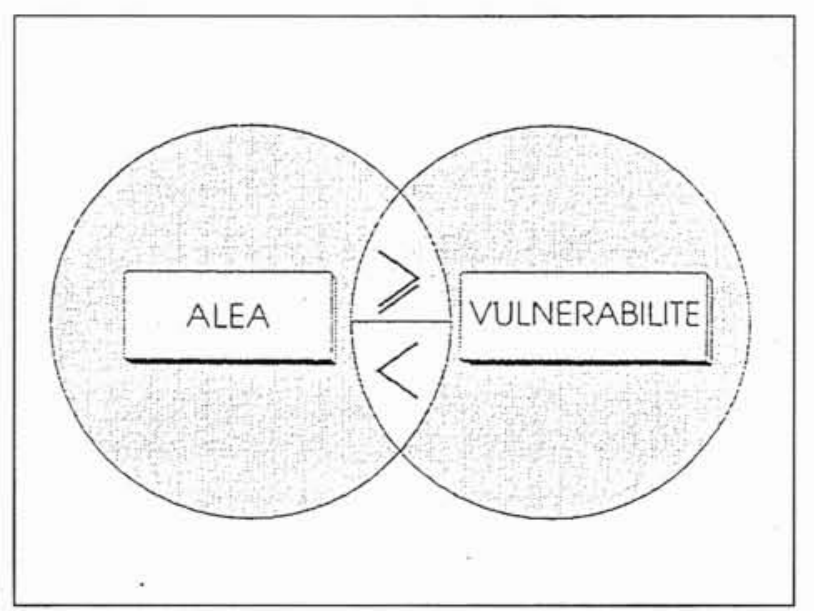

2. Conceptualisation du risque. 


\section{III. - FONCTION HYDRAULIQUE}

Dans ce paragraphe nous allons tenter de mettre en évidence les différentes fonctions hydrauliques que peuvent jouer les zones d'épandage des crues. Il est évidemment difficile d'analyser dans le détail la complexité de leur fonctionnement dans un contexte hydrologique présentant lui-même une grande complexité. En effet, les conséquences hydrauliques traduisent en fait un déplacement dans le temps ou l'espace d'un certain volume d'eau apporté par un épisode pluvieux. Or, il n'existe pas de crue type unique pour un fleuve comme la Loire, mais tout un régime hydrologique avec des crues courantes et des crues exceptionnelles, des crues plus ou moins brutales en fonction des événements météorologiques générateurs (crues cévenoles du haut bassin versant et crues océaniques du bassin versant aval)... Il est évident que l'impact d'une zone d'épandage de crue dépend du rapport entre son volume mobilisable et celui de la crue qui passe. Cependant il est possible de mettre en évidence de grands principes généraux et l'efficacité de tels aménagements résulte autant de leur nombre et de leur répartition le long du cours du fleuve que de l'efficacité réelle de chacun d'entre eux.

\subsection{Laminage}

L'effet de laminage (fig. 3), bien connu des hydrauliciens, traduit le phénomène de stockage-déstockage de l'eau dans un certain volume rempli par une inondation. Lors d'une crue, l'augmentation du débit dans une section de rivière se traduit par une augmentation du niveau de l'eau, nécessaire à l'augmentation simultanée de surface mouillée et de pente hydraulique pour respecter les lois fondamentales de I'hydraulique que sont la conservation de la masse et celle de la quantité de mouvement. Les équations de Barré de Saint-Venant couramment utilisées pour représenter le fonctionnement hydraulique d'un cours d'eau, permettent de modéliser ces lois physiques. Pour que le niveau d'eau puisse monter, il faut utiliser une partie du volume d'eau arrivant pour remplir la zone « inondable" proche où le niveau d'eau est directement lié à celui atteint dans le lit principal de la rivière.

Il en résulte une déformation de l'hydrogramme de crue avec une diminution du débit instantané maximum et une augmentation de sa durée caractéristique, le volume total restant constant. Ces deux modifications sont celles qui vont nous intéresser principalement pour leur incidence sur la propagation de la crue à l'aval. On comprend aisément l'intérêt de réduire l'intensité maximale de l'événement : la contrainte hydraulique sera réduite d'autant à l'aval d'une zone d'écrêtement de crue améliorant l'efficacité des moyens de protection que l'on peut être amené à y mettre en place. L'augmentation de la durée de l'hydrogramme peut aussi avoir des conséquences favorables, en particulier sur l'aspect « ressource en eau » que nous allons analyser dans la paragraphe suivant.

\subsection{Ressource en eau}

Le rôle des zones d'épandage des crues sur la ressource en eau est lié à deux facteurs différents : d'une part la durée des hautes eaux, conformément à l'augmentation de la durée des hydrogrammes, et d'autre part la surface d'échange entre les eaux de surface et les eaux souterraines.

Le paramètre durée influe directement sur la durée d'échange entre le lit mineur du cours d'eau et la nappe qui l'accompagne. On sait, suite à différentes études sur les échanges nappes-rivières [3] que ceux-ci sont généralement très importants au travers des berges, où la vitesse habituelle du courant empêche (ou limite) les phénomènes de colmatage. Une augmentation de la durée des écoulements à plein bord se traduit donc en première approximation par une augmentation proportionnelle des volumes d'eau infiltrés. La ressource en eau mobilisable dans la nappe s'en trouve donc augmentée d'autant. Si vous multipliez par deux les durées caractéristiques des crues (fig. 4), vous multipliez par deux le volume d'eau disponible dans la période qui suit. En dehors de toute exploitation directe de la nappe d'eau, cela peut diminuer les problèmes d'étiages.

L'importance des échanges entre nappes souterraines et champs d'expansion des crues est plus difficile à mettre en évidence. En effet. ces zones sont souvent le siège de dépôt des matières fines transportées en suspension par les écoulements en crue, et ces particules fines ont tendance à colmater les sols et à en diminuer la perméabilité. Cependant l'importance des surfaces concernées lorsque ces zones sont étendues compense peu ou prou cet effet réducteur et on peut donc penser, sans qu'il soit aisé de le quantifier précisément, que la remise en eau de grandes surfaces pour l'épandage des crues aura un effet positif sur la ressource en eau mobilisable.

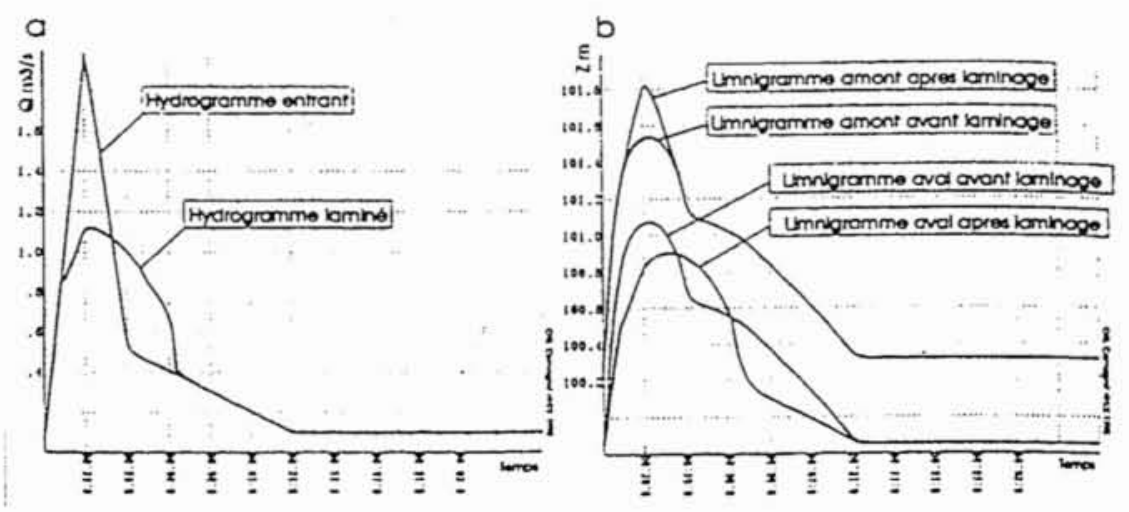

3. Le phénomène du laminage. 


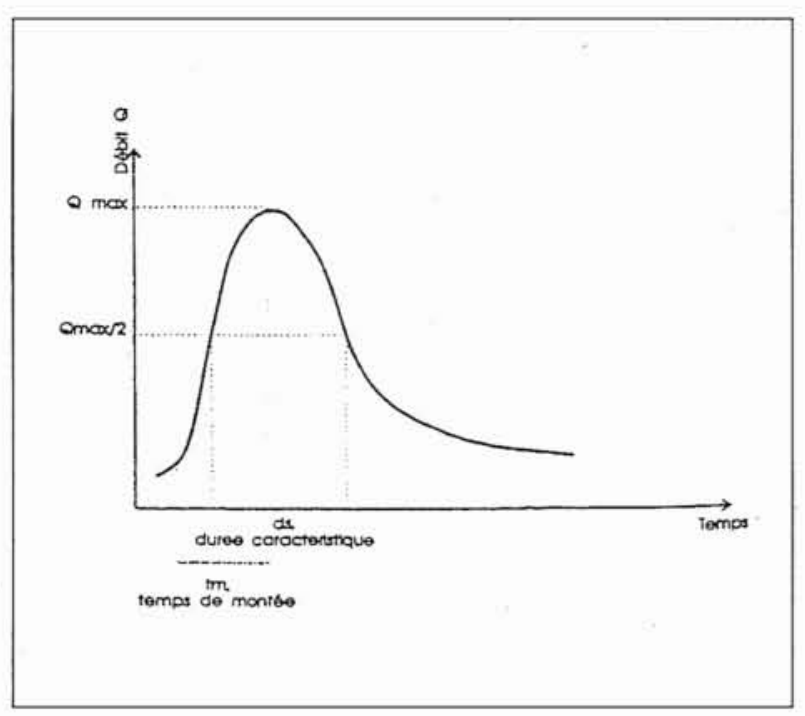

4. Durée caractéristique de crue.

\section{- 3.3 Equilibres naturels}

Par ce rôle hydraulique, ces zones peuvent contribuer au maintien de certains équilibres naturels importants pour la pérennité de certains ouvrages. Les processus géomorphologiques et les transports solides associés aux écoulements turbulents entrainent des interactions complexes entre l'eau et le substrat sur lequel elle coule [4]. On connaît les déséquilibres qui peuvent apparaître suite à des aménagements n'ayant pas pris ces problèmes en considération : extractions de matériaux, redimensionnement de lit, endiguement généralisé... entraînant l'incision des lits, l'érosion des berges... Le maintien de vastes zones inondables, favorisé aussi par des lits mineurs non surdimensionnés, a pour effet de limiter les impacts d'autres aménagements ponctuels en réduisant les intensités et les vitesses maximales. L'équilibre entre l'écoulement et le substrat est ainsi plus facile à atteindre et les processus de dissipation d'énergie se produisent plus naturellement. En se rapprochant ainsi des équilibres naturels, on prévoit une nette diminution des charges d'entretien sur les infrastructures hydrauliques proches et une plus grande pérennité de ces ouvrages.

De la même façon, et pour des raisons un peu similaires liées au ralentissement des écoulements dans ces zones, on observe des effets auto-épurateurs dans ces milieux ralentis où un certain nombre de produits polluants transportés par l'eau peuvent soit se déposer (piégés par les sédiments en suspension) soit se dégrader (en raison du temps de séjour dans un milieu particulier) [5]. Ces processus auto épurateurs permettent d'améliorer la qualité générale de l'eau et, en conséquence, de la rendre plus facilement mobilisable pour les besoins de consommation. Il va sans dire que cela n'empêchera pas de prendre des mesures de dépollution à la source, mais cela peut contribuer à atténuer l'effet de certaines pollutions résiduelles ou occasionnelles en évitant une détérioration continue de la qualité.

\section{IV. [ FONCTION ÉCONOMIQUE}

Bien que les zones utilisées pour l'écrêtement des crues soient soumises à une contrainte hydraulique assez forte, en comparaison des zones destinées à l'urbanisme, il est possible de leur faire jouer un rôle économique producteur de richesse. La contrainte hydraulique peut en effet y être analysée grâce aux études hydrologiques et hydrauliques, et leur caractère inondable ne se traduit pas par une mise en eau continue ni même forcément annuelle. Il peut s'agir de zones qui ne seront inondées que par des crues de périodes de retour pluriannuelles sans pour autant réduire leur efficacité pour le laminage des crues plus rares, objectif que l'on recherche habituellement pour la préservation des zones urbaines en particulier.

\subsection{Mise en valeur agricole}

Les fonds de vallées alluviales qui peuvent servir de zone d'épandage temporaire des crues sont généralement constitués de sols potentiellement riches pour une mise en valeur agricole du fait des processus à l'origine de leur formation : dépôts de limons...

Par ailleurs, il existe un certain nombre de spéculations agricoles qui tolèrent relativement bien une contrainte hydraulique importante [6]. Les surfaces toujours en herbe, en particulier, utilisées pour l'élevage ou pour la production de fourrage et en constante augmentation depuis quelques années (à l'échelle de la France) [7] tolèrent facilement d'être submergées de quelques jours à quelques semaines par an sans perte de rendement. Cela nécessite bien entendu que la saison de submersion la plus fréquente ne coïncide pas avec la saison d'utilisation agricole de ces surfaces, ce qui est généralement le cas (crues d'hiver et période de pâturage en été).

Outre les prairies, il existe d'autres productions qui tolèrent des submersions limitées: arboriculture, cultures pérennes, etc. Chacune a ses contraintes propres en terme de saison d'inondation, de profondeur d'eau admise et de durée de submersion [6]. Il importe donc de les implanter en connaissant le mieux possible la contrainte hydraulique subie. Par exemple, la populiculture (culture de peupliers) permet de mettre en valeur des sols fortement hydromorphes sans difficultés particulières.

Il est nécessaire d'accompagner un tel usage des sols par des mesures de compensation pour les occasionnelles pertes de production liées à ce rôle hydraulique que l'on voudrait leur faire jouer : assurances, indemnités pour perte de récolte, travaux d'assainissement complémentaires pour faciliter le retrait des eaux et limiter les durées d'inaccessibilité, etc. peuvent compenser cette servitude hydraulique. Il est certain que les moyens de négociations avec la profession agricole existent et devraient permettre de maintenir une mise en valeur économique de ces zones tout en maintenant leur caractère inondable. L'évolution du monde agricole liée à la nouvelle Politique Agricole Commune devrait même faciliter un tel processus.

\subsection{Mise en valeur touristique}

Outre la fonction productrice directe, les zones de bordure de cours d'eau, et les zones inondables en particulier, peuvent jouer un rôle important dans le développement touristique de certaines micro-régions. Les paysages associés aux cours d'eau sont particulièrement attrayants pour un tourisme vert si l'on fait l'effort de maintenir leur qualité Forêts alluviales et friches humides sont autant de lieux qu peuvent attirer des citadins à la recherche d'une nature absente de leur zone d'habitation et la présence de l'eau proche rend ces sites encore plus attractifs.

Ces zones peuvent aussi permettre de développer des infrastructures de loisirs pour peu que l'on tienne compte dans leur conception de la présence occasionnelle de l'eau : terrains de jeu, terrains de camping, terrains de sports peuvent fort bien supporter en dehors de la saison de grande uti- 
lisation (printemps-été) une submersion temporaire au prix de quelques aménagements adaptés. La figure 5 en montre un exemple.

De tels développements en zone inondable permettent de les valoriser économiquement sans les empêcher de conserver un caractère inondable utile à la protection de zones plus vulnérables à l’aval.

\section{V. 圆 RÔLE ÉCOLOGIQUE}

Les zones humides connexes au cours d'eau, parmi lesquelles certaines zones potentielles d'épandage des crues, sont réputées pour leur potentiel écologique et leur richesse biologique. Plusieurs fonctions écologiques sont associées à ces zones que nous allons brièvement passer en revue.

\subsection{Frayères}

Les prairies inondées de bord de rivière sont le lieu de reproduction de certaines espèces de poisson comme le brochet. Il faut pour ce faire une submersion saisonnière qui permette l'accès des reproducteurs et le développement des pontes grâce à des conditions physiques (vitesses de courant. hauteur d'eau) adaptées aux besoins de ces espèces. Le renouvellement de la faune est une condition nécessaire au maintien d'un environnement de qualité et favorise aussi les activités de loisirs comme la pêche dont on connât l'importance en France et sur les bords de la Loire en particulier.

\subsection{Ripisylve}

Pas directement liée aux zones d'épandage des crues, mais potentiellement présente le long de celles-ci, la ripisylve correspond à la végétation de la bande rivulaire proche du lit mineur d'un cours d'eau. C'est un milieu spécifique tant par la flore qui s'y développe que par la faune qu'elle abrite. Il joue un rôle particulier dans le maintien de l'écosystème aquatique en apportant des matières organiques, en régulant la température du milieu aquatique grâce à l'ombre portée, et en fournissant des habitats à la faune aquatique (racines) et à la faune terrestre [8]. On comprend que cela participe au maintien d'un environnement riche.

Sous réserve, là où cela est vraiment nécessaire, d'un entretien minimum régulier, une ripisylve ne risque guère de créer des problèmes particuliers, et même le risque d'embâcle souvent avancé, est rarement objectivement analysé. La présence d'une ripisylve continue peut même contribuer à le diminuer puisque l'on constate qu'elle piège souvent une partie des matériaux flottants transportés par le cours d'eau.

\subsection{Forêts alluviales}

Les forêts alluviales qui se développent généralement dans des zones au caractère inondable marqué jouent aussi un rôle écologique important dû à la spécificité de la végétation qui les compose et au milieu particulier qu'elles constituent. Leur très faible vulnérabilité à des inondations même prolongées les rend particulièrement adaptées dans les sites où la contrainte hydraulique est la plus forte. En dehors de ces périodes de submersion, elles constituent des sites potentiellement attrayants pour des randonneurs des classes vertes, etc... et contribuent ainsi à l'équilibre écologique et social.

\subsection{Zones humides}

Existent aussi les « zones humides " même temporaires qui peuvent jouer un rôle d'accueil de certains oiseaux migrateurs, par exemple, en raison de la spécificité du milieu naturel qui s'y développe. La présence d'une faune diversifiée est un attrait pour les promeneurs et les touristes et intéresse le développement touristique d'une région. A ce titre il semble intéressant de maintenir ici et là des zones de ce type de manière pérenne et de développer autour les infrastructures nécessaires à l'accueil du public tout en respectant la qualité naturelle du lieu considéré.

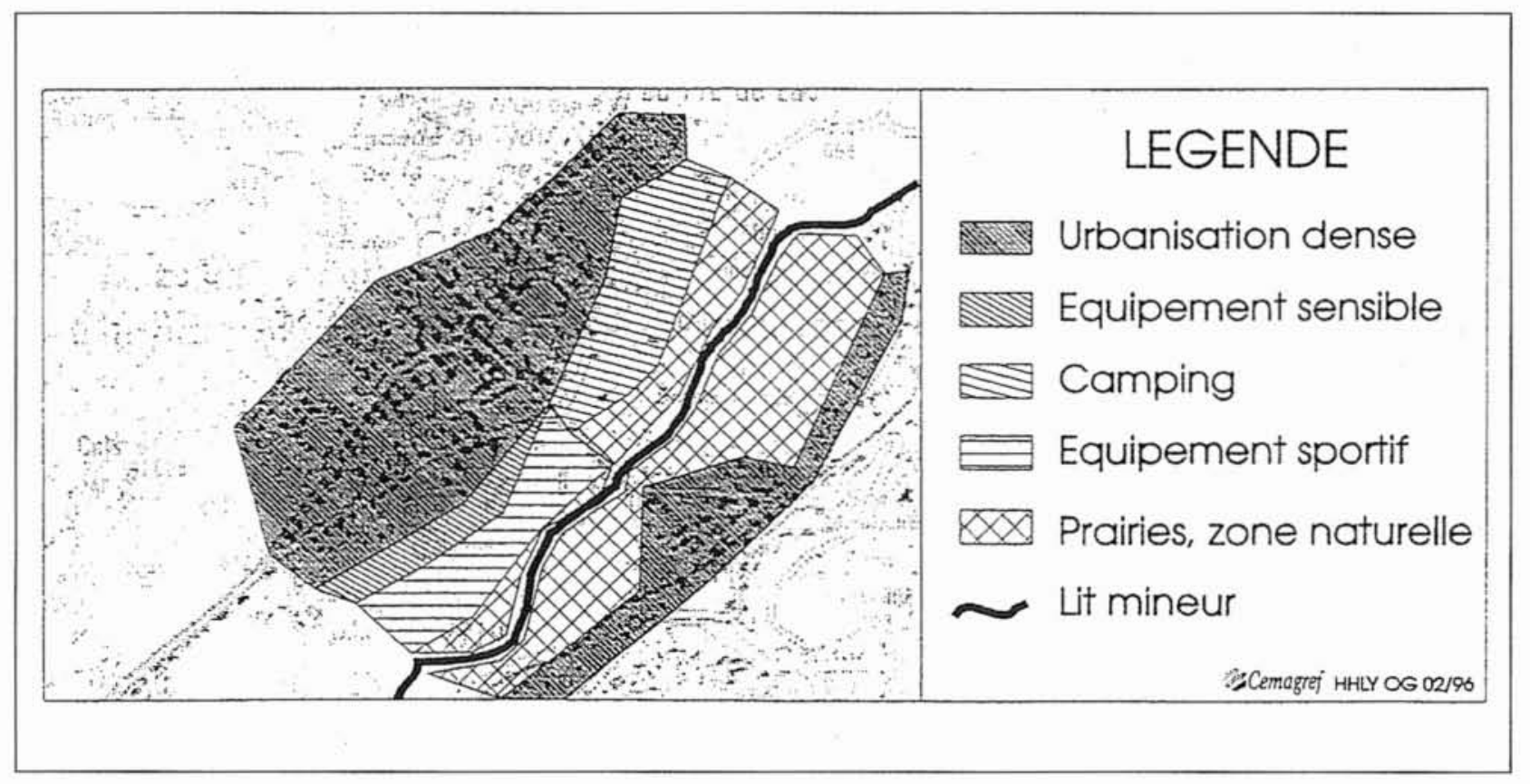

5. Exemple d'occupation de l'espace en bordure d'une rivière. 


\section{E VERS UNE GESTION PLUS EFFI- CACE DES ZONES INONDABLES}

On a vu dans les paragraphes qui précèdent les multiples rôles que peuvent jouer les zones inondables tant sur le plan hydraulique que sur le plan économique et écologique. L'intensification de l'agriculture depuis les années 50 et l'évolution de l'urbanisme ont créé une forte pression sur ces zones facilement accessibles des vallées alluviales et ont conduit à une politique d'aménagement parfois oublieuse de certaines de ces fonctions.

Aujourd'hui, l'évolution économique et sociale de la société [9] nous amène à réviser la manière de gérer ces espaces et il convient de se doter des méthodes et des outils qui permettent une meilleure analyse des différents enjeux et une gestion équilibrée et durable des vallées.

\subsection{Limites des solutions classiques}

Les solutions classiques, qui font appel à des aménagements structurels de type endiguements et recalibrages, ont atteint leur limite en terme d'efficacité en particulier en raison du manque de diversification dans le choix et le dimensionnement de ceux-ci. C'est ainsi, si l'on prend l'exemple de la Loire, que l'on se retrouve avec un endiguement continu et assez uniforme qui protège indifféremment zones rurales et zones urbaines, zones d'activité et zones de loisirs. Quelques déversoirs ont bien été installés depuis longtemps pour préserver le caractère inondable de certains vals, mais le niveau de protection retenu est de l'ordre de la crue centennale [2], c'est-à-dire plus proche d'un objectif «zone urbaine » que d'un objectif «zone rurale». Il est donc à la fois difficile d'y maintenir une "culture" de l'inondation et d'y éviter une évolution de l'usage des sols aux conséquences potentiellement dommageables.

Cette relative uniformisation de la protection, associée à la menace de ruptures des digues en cas de fortes crues (erreur de conception, entretien insuffisant), a pour conséquence l'impossibilité de prévoir quelles zones seront touchées en cas d'événement exceptionnel qui dépasserait le niveau de dimensionnement de ces ouvrages. De plus, la généralisation à tout le linéaire de ces solutions classiques limite intrinsèquement leur efficacité : d'un côté on réduit la probabilité du débordement (à régime hydrologique constant) et de l'autre on supprime toutes les zones d'épandage de crue, ce qui entraîne une aggravation des pics de crue (fig. 3), ce dont ont pris conscience les aménageurs depuis longtemps sans cependant en tirer toutes les conséquences opérationelles. Il est possible que cela aboutisse in fine à une aggravation de l'aléa plutôt qu'à sa diminution. Comme de plus on maitrise mal l'évolution de la vulnérabilité de ces zones, comme en témoigne l'urbanisation de certains vals le long de la Loire, on aboutit en fait à une augmentation objective du risque analysé collectivement et globalement.

Or on a vu que la mise en valeur des zones réputées inondables pouvait faire appel à des solutions très diversifiées, depuis la forêt alluviale jusqu'à la zone de loisirs en passant par une mise en valeur agricole elle-même potentiellement diversifiée. Aujourd'hui, il convient d'utiliser ce potentiel de diversité pour mieux équilibrer le développement de ces zones et surtout améliorer la sécurité des zones les plus sensibles et réduire les conséquences dommageables d'une crue exceptionnelle dont la probabilité est loin d'être nulle.

\subsection{Concepts du ralentissement dynamique}

Pour répondre à cette nécessité de maîtrise du risque, il convient de se placer dans un nouveau cadre conceptuel pour définir les aménagements pertinents. Les solutions tra- ditionnelles font presque toujours appel à une accélération des transits vers l'aval : à l'exception des barrages, endiguements et recalibrages vont dans ce même sens. Nous proposons aujourd'hui d'inverser cette tendance et de faire appel à la règle d'or du Ralentissement Dynamique [10], qui consiste à l'inverse, à freiner l'eau partout où cela est possible mais uniquement aux périodes les plus critiques, c'està-dire au moment des pointes de crue, pour éviter une stérilisation de l'espace. Remarquons que ce n'est pas une idée nouvelle puisque déjà au XIX $\mathrm{X}^{\mathrm{e}}$ siècle le père de l'hydraulique fluviale, M. De Saint-Venant présentait ces idées devant l'Académie des Sciences [1I].

Cette idée amène à préconiser une véritable maîtrise de l'occupation des sols dans les zones inondables permettant de leur attribuer un niveau de protection plus raisonnable et ainsi de favoriser leur fonction d'épandage des crues sans pour autant leur ôter toute fonction économique. Il convient d'assortir cette contrainte hydraulique de mesures d'accompagnement (assurances, indemnisations, contreparties matérielles) destinées à mettre en œuvre une réelle solidarité entre zones. Et cela devrait être mis en œuvre en particulier dans les vals équipés dès le XIX ${ }^{\mathrm{C}}$ siècle de déversoirs. Ces idées générales se retrouvent dans les derniers textes issus du ministère de l'Environnement (circulaire de novembre 1994, loi Environnement et PPR) ainsi que dans le rapport parlementaire de 1994 [12].

\subsection{Existence d'outils de modélisation}

Pour mettre en pratique ces concepts, il existe aujourd'hui des outils de modélisation dont certains ont été validés sur le bassin versant de la Loire [13]. La méthode Inondabilité [14], [15] du Cemagref a été développée dans ce but : asseoir les

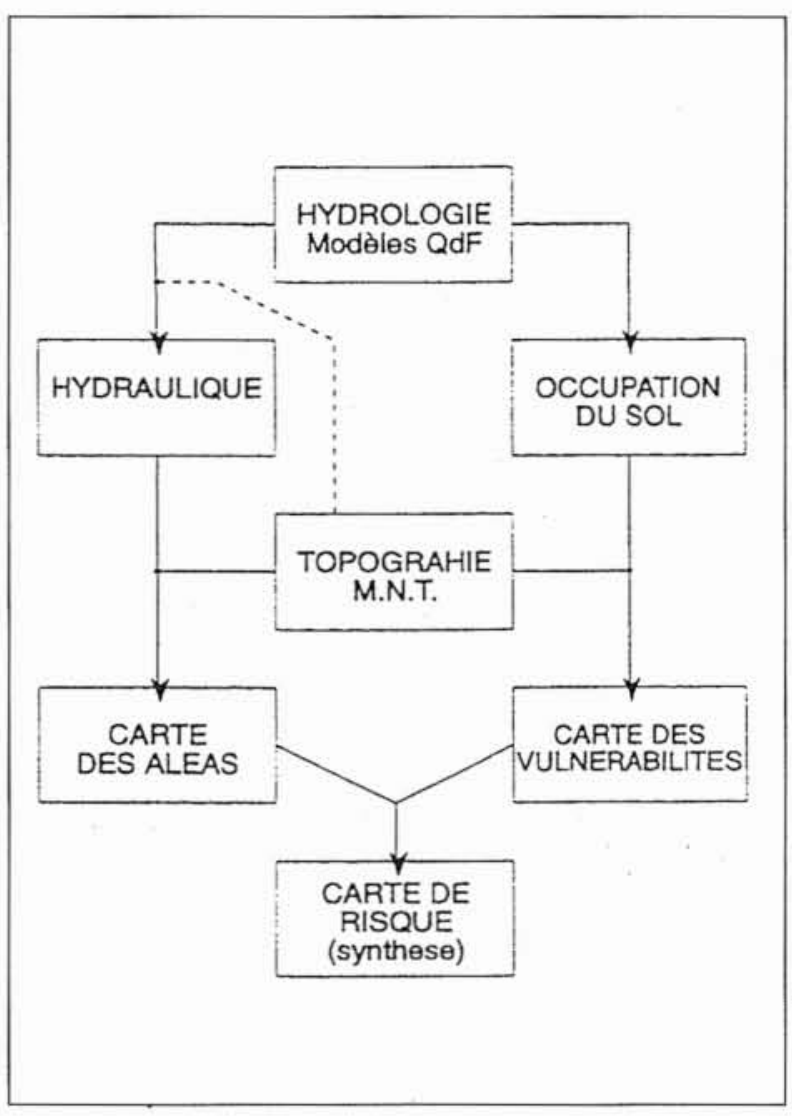

6. Organigramme simplifié de la méthode INONDABILITÉ. 
bases objectives d'une analyse diversifiée du risque d'inondation le long d'un cours d'eau et permettre une véritable négociation entre les différents acteurs présents autour de ces systèmes. Cette méthode permet une quantification compatible des deux composantes du risque que sont l'aléa et la vulnérabilité, et la traduction sous forme d'une cartographie de synthèse des résultats de ces études techniques de manière à les rendre plus facilement compréhensibles par l'ensemble des personnes concernées.

Dans une telle représentation, tous les enjeux liés au milieu aquatique sont représentables : ressource en eau, milieu naturel, potentiel économique... On dispose alors des bases objectives d'une contractualisation de la gestion de l'espace entre les différents acteurs concernés, où les intérêts diversifiés et parfois contradictoires des différents acteurs peuvent s'exprimer. Reste ensuite à négocier les indispensables compromis et mesures d'accompagnement qui permettront une mise en valeur plus équilibrée de ces milieux dont on espère en particulier une meilleure maîtrise du risque, et à tout le moins, des dommages qui en résultent.

\section{VII. $\mathrm{B}$ CONCLUSION}

Les zones d'épandages de crue peuvent jouer un rôle considérable pour divers enjeux liés à l'aménagement des rivières. L'émergence de nouveaux concepts d'aménagement, dont la mise en œuvre est facilitée par les progrès scientifiques dans ce domaine et par de nouvelles méthodes comme Inondabilité, doivent permettre un développement diversifié, harmonieux et durable des vallées alluviales en favorisant le maintien d'une activité économique adaptée et la préservation de zones naturelles à fort potentiel écologique.

Ces réflexions adaptées au cas de la Loire se retrouvent quasiment à l'identique dans des contextes très différents comme le bassin du Mississippi [16] où les analyses menées suite à la grande crue de 1993 ont amené les décideurs aux mêmes conclusions : il faut mettre en œuvre de nouveaux concepts pour des aménagements plus diversifiés des plaines d'inondation permettant de maintenir un fonctionnement plus équilibré et plus proche des équilibres naturels si l'on veut éviter à la fois une augmentation inconsidérée des dommages liés aux inondations (exceptionnelles) et la préservation d'un environnement de qualité dont les bénéfices indirects, socialement de plus en plus reconnus, peuvent être très importants.

Il est urgent de relever ce défi de l'aménagement sur le terrain lui-même et d'utiliser les résultats scientifiques disponibles concernant la connaissance de ces milieux et de leur fonctionnement, sous peine d'être accusé demain d'avoir omis d'appliquer le "principe de précaution" $\mathrm{et}$ laissé se développer des aménagements coûteux et inefficaces qui ne répondront pas aux exigences de la société future.

\section{BIBLIOGRAPHIE}

[1] Chastan B., Gilard O., Givone P., Oberlin G. (1995). La prise en compte du risque d'inondations Ingênieries. EAT, $n^{\circ} 2$, juin 1995, 13-20.

[2] DiREN, Service de Bassin Loire Bretagne (1988). - Protection de la Loire moyenne contre les crues, anonyme, décembre 1988 .
[3] ILLE C. (1992), - Interface rivière-nappe : caractérisation physique et chimique; rôle protecteur de laquifere; cas de l'île du Grand Gravier (69). Thèse de doctorat spécialité Mécanique, Université J. Fourier Grenoble I, février 1992. 155 p. + ann.

[4] RAMEz P. (1995). - Erosion et transport solide en rivière: Tome 1: Guide pour la compréhension des phénomènes. CEMAGREF, Dicova. Série Etudes: Gestion des milieux aquatiques, $130 \mathrm{p}$.

[5] DE LANEY T.A. (1995). - Benefits to downstream flood attenuation and water quality as a result of constructed wetlands in agricultural landscapes. Journal of soil and water conservation, nov.-dec. 1995, pp. 620-626.

[6] Desbos E. (1995), - Qualification de la vulnérabilité du territoire face aux inondations, D.E.A. "Conception en Bâtiment et techniques urbaines ». INSA Lyon, Laboratoire Méthodes, $70 \mathrm{p}$.

[7] Ripoche A., Agence de l'eau Loire Bretagne (1995). Influence de l'occupation des sols sur les inondations, agence de l'eau Loire-Bretagne, 1995.

[8] MARIdet L., Colin M.-P. (1995), - La végétation aux abords des rivières: source de vie et d'équilibre. Plaquette éditée par le Ministère de l'Environnement, 1995.

[9] FABIANI J.-L.. THEYS J. (1987), - La société vulnérable, évaluer et maîtriser les risques, Presses de l'école normale supérieure, 1987.

[10] OBERLIN G. (1994), - Contribution à une gestion intégrée du patrimoine des eaux continentales... par une protection raisonnée et négociée contre les inondations. Journée du Palais d'Iéna «Des aménagements hydrologiques, pour répondre à la diversité des objectifs économiques, sociaux et écologiques de notre époque ", Académie des Sciences, CADAS, 26 mai 1994.

[11] De Saint-Venant (1846), - Mémoire sur la dérivation des eaux pluviales qui entraînent les terres et des sols en pente et qui inondent les vallées, présenté à l'Académie des Sciences, le 20 avril 1846, repris in Environnement Magazine, 1543, décembre 1995.

[12] Mathot P., Mariani T. (1994). - Inondations : une réflexion pour demain, rapport $n^{*} 1641$ de l'Assemblée Nationale, rapport de la commission d'enquête parlementaire sur les causes des inondations et les moyens d'y remédier.

[13] Sourisseau J., Galea G. (1996), - Représentativité des modèles $Q d F$ : application à la régionalisation des régimes de crue du bassin versant de la Loire. Cemagref, 53 p. + annexes.

[14] Gautier J.-N. (1991), - Inondabilité, cartographie de synthèse. Rapport $\mathrm{X}^{\mathrm{c}}$ Plan Etat-Région Rhône-Alpes. risque naturels en montagne, crues et inondations en vallée, $1991,100 \mathrm{p}$.

[15] Cemagref hHLy. - Guide pratique de la méthode Inondabilité, à paraître avec le concours de l'interagences de I'Eau, 1996.

[16] RASMUSSEN J. L. (1994), - Floodplain management into the 21st century: a blueprint for change - sharing the challenge, 1994, Water International, 19 (1994), pp. 166176. 